Article

\title{
Development of a Direct Headspace Collection Method from Arabidopsis Seedlings Using HS-SPME-GC-TOF-MS Analysis
}

\author{
Miyako Kusano ${ }^{1,2, *}$, Yumiko Iizuka ${ }^{1,3}$, Makoto Kobayashi ${ }^{1}$, Atsushi Fukushima ${ }^{1}$ and \\ Kazuki Saito ${ }^{1,4}$
}

1 RIKEN Plant Science Center, Tsurumi, Yokohama 230-0045, Japan; E-Mails: kobamako@riken.jp (M. Kobayashi); a-fukush@psc.riken.jp (A.F.)

2 Department of Genome System Sciences, Graduate School of Nanobioscience, KIHARA Institute for Biological Research, Yokohama City University, Totsuka, Yokohama 244-0813, Japan; E-Mail: mkusano005@psc.riken.jp (M.K.)

3 Department of Genome System Science, Graduate School of Nanobioscience, Yokohama city University, Kanazawa, Yokohama 236-0027, Japan; E-Mail: yiizuka-ri@psc.riken.jp (Y.I.)

4 Department of Molecular Biology and Biotechnology, Graduate School of Pharmaceutical Science, Chiba University, Chuo, Chiba 260-8675, Japan; E-mail: ksaito@psc.riken.jp (K.S.)

* Author to whom correspondence should be addressed; E-Mail: mkusano005@psc.riken.jp; Tel.: +81-45-503-9442; Fax: +81-45-503-9489.

Received: 20 February 2013; in revised form: 21 March 2013 / Accepted: 26 March 2013 / Published: 9 April 2013

\begin{abstract}
Plants produce various volatile organic compounds (VOCs), which are thought to be a crucial factor in their interactions with harmful insects, plants and animals. Composition of VOCs may differ when plants are grown under different nutrient conditions, i.e., macronutrient-deficient conditions. However, in plants, relationships between macronutrient assimilation and VOC composition remain unclear. In order to identify the kinds of VOCs that can be emitted when plants are grown under various environmental conditions, we established a conventional method for VOC profiling in Arabidopsis thaliana (Arabidopsis) involving headspace-solid-phase microextraction-gas chromatography-time-of-flight-mass spectrometry (HS-SPME-GC-TOF-MS). We grew Arabidopsis seedlings in an HS vial to directly perform HS analysis. To maximize the analytical performance of VOCs, we optimized the extraction method and the analytical conditions of HP-SPME-GC-TOF-MS. Using the optimized method, we conducted VOC profiling of Arabidopsis seedlings, which were grown under two different nutrition conditions, nutrition-rich and nutrition-deficient conditions. The VOC profiles clearly
\end{abstract}


showed a distinct pattern with respect to each condition. This study suggests that HS-SPME-GC-TOF-MS analysis has immense potential to detect changes in the levels of VOCs in not only Arabidopsis, but other plants grown under various environmental conditions.

Keywords: solid-phase microextraction; HS-SPME-GC-TOF-MS; volatile organic compounds; VOC profiling; Arabidopsis

\section{Introduction}

Plants and animals, including humans and microorganisms, can produce volatile organic compounds (VOCs). For example, VOCs in exhaled breath show specific profile patterns with respect to various cancers [1-8]. In vitro and in vivo experiments have been conducted for VOC profiling in rat and cattle breath as case studies of animal VOCs $[9,10]$. Microorganisms can produce various types of VOCs, which are likely to have crucial effects on soil ecology [11] and on interactions across other organisms [12-15]. Plants also contain a large variety of VOCs, including simple gases and other VOCs. At present, approximately 1,700 compounds are identified as VOCs, and these account for more than $1 \%$ of plant secondary metabolites [16,17]. The main classes of plant VOCs are terpenoids, phenylpropanoids/benzenoids and fatty acid and amino acid derivatives [18]. Plants can emit various types of VOCs, not only to communicate with herbivores, microbes and neighboring plants, but also to respond to abiotic factors, such as light and/or temperature, atmospheric $\mathrm{CO}_{2}$ concentration or nutrition [19-21]. Therefore, plant VOCs are potential biomarkers capable of identifying the physiological states of plants and the stresses to which they have been subjected.

Sensitive methods have been developed for headspace (HS) sampling and VOC analysis. HS sampling is a non-destructive method for collecting volatile compounds. Static and dynamic sampling methods are widely used for collecting HS samples [22]. Static HS sampling is suited for qualitative analyses of VOCs and surveys of VOC profiles of different plant species or cultivars at a single time point, while dynamic HS sampling enables us to quantify changing VOC emissions in the form of real-time analysis. Many devices can be used to collect VOCs, e.g., solid phase microextraction (SPME) fibers, stir bar sportive extraction (SBSE) devices [23,24] and in-tube extraction (ITEX) [25,26]. Sensor arrays composed of thin nanostructure films, such as monolayer-capped metallic nanoparticles and chemiresistive random network of single-walled carbon nanotubes, can be used as diagnostic tools to detect VOCs in exhaled breath samples [27,28].

Gas chromatography (GC)-based techniques are widely used for static HS analyses, including GC-mass spectrometry (GC-MS), GC-flame ionization detection (GC-FID), GC-electroantennogram detection (GC-EAD) and two-dimensional GC-MS (GC×GC-MS) [17,22,29]. We can directly inject a mixture of VOCs into GC instruments, since no derivatization steps are required. Fast isoprene sensor chemiluminescence detection (FIS), laser-based infrared photoacoustic (PA) spectroscopy and proton transfer reaction-mass spectrometry (PTR-MS) enabled us to conduct real-time detection of VOCs from plants [18].

One ultimate goal of metabolomics is to identify and quantify the metabolome in organisms, which includes VOCs. Development of MS-based technologies in metabolomics enables the measurement of 
hundreds of metabolites [30-32]. In particular, electron impact-time-of-flight-MS (EI-TOF-MS) provides us with comprehensive information on molecular fragments as mass-to-charge ratios [33]. Annotation of each spectrum of a VOC is performed by finding an exact match to a reference spectrum from well-established libraries, such as NIST/EPA/NIH Mass Spectral Library [34], Adams Library [35], Terpenoids Library (http://massfinder.com/wiki/Terpenoids_Library) and VocBinbase [36]. The Kovats retention index (RI), calculated by comparing the chromatographic retention time of $n$-alkane peaks, is often used to annotate detected peaks [37]. A combination of these libraries and RI information is indispensable to annotate detected peaks in a non-targeted matter. So far, HS-SPME-GC-MS analysis has been applied to the comprehensive VOC profiling of apple fruits [38], tomato flavors [39,40], human nevi and melanoma [6] and fungus extracts [41].

The VOCs emitted from the flowers and foliage of Arabidopsis thaliana (Arabidopsis), which is widely used as a model species for studying plant biology, have been investigated [42-45]. However, many studies were focused on the VOCs induced by attacking herbivores [46-48] and microorganisms [49,50]. We hypothesized that the composition of VOCs may differ when grown under different nutrient conditions, i.e., macronutrient-deficient conditions. However, the relationships in the plants between macronutrient assimilation, metabolic networks and VOC composition in these plants remain unclear.

To obtain insights into these relationships, we developed a way of VOC profiling by using HS-SPME-GC-TOF-MS (Figure 1). First, Arabidopsis seedlings were directly grown in HS vials. Volatile compounds in each vial were collected using an appropriate SMPE fiber and analyzed by using HS-SPME-GC-TOF-MS in a non-targeted manner. After data processing and normalization as described by Kusano et al. [51], we conducted statistical analysis on the dataset. We performed VOC profiling in the HS of Arabidopsis seedlings grown under two nutrient conditions as a case example. Distinct patterns of VOC profiles were found by applying the optimized analytical method. The annotated VOCs were determined by performing multivariate analysis and $t$-tests as discriminative parameters between the two conditions.

Figure 1. Volatile organic compound (VOC) profiling of Arabidopsis seedlings directly in headspace (HS) vials. Abbreviation: EDTA, ethylene-diamine-tetra-acetic acid.

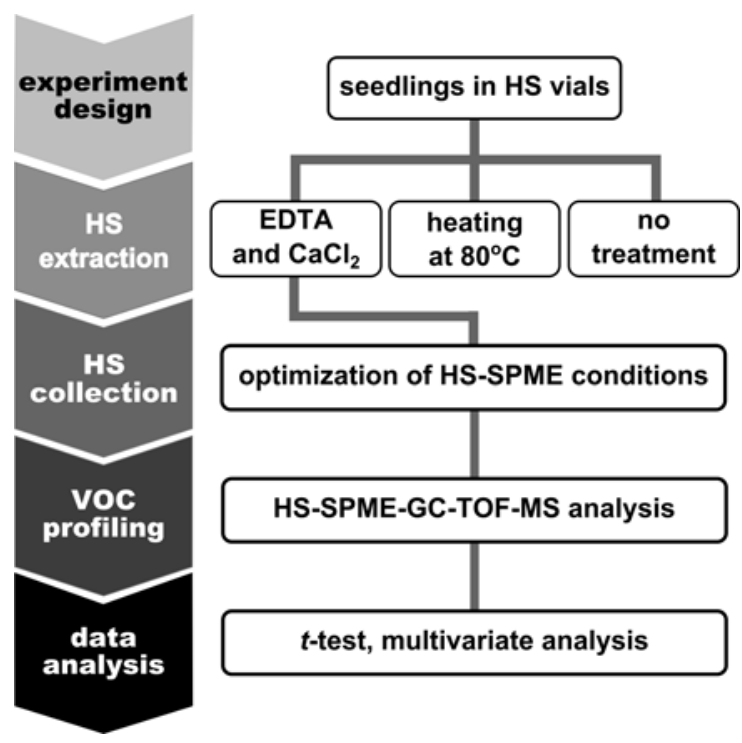




\section{Results and Discussion}

\subsection{Experiment Design for VOC Profiling in the HS of Arabidopsis Seedlings}

Before starting the experiment, we performed HS-SPME-GC-TOF-MS to confirm that the growth medium and water for cultivating Arabidopsis seedlings contained no volatile substances (data not shown). We grew Arabidopsis seedlings in a closed HS vial in order to conduct HS analysis directly (Figure 1a). Arabidopsis seedlings in sterile liquid culture containing Murashige and Skoog medium with $1 \%$ sucrose (MS condition, hereafter) grew better compared to those in sterilized Milli-Q water (water condition, hereafter, Figure 2a). Seedlings grew 10-20 times larger under the MS condition than water, in terms of dry weight (Figure $2 b$, blue bar). We next evaluated the effect on $\mathrm{CO}_{2}$ content toward plant growth $\left(\mathrm{CO}_{2}\right.$ depletion inhibits plant growth) by weighing the dry matter from Arabidopsis seedlings grown in an HS vial, which was closed with a magnetic screw cap equipped with silicon/polytetrafluoroethylene septa (PTFE) or affixed with MilliSeals. There were no drastic effects toward growth whether we used PTFE or MilliSeal seals (Figure 2b). Thus, we expected that changes in the VOC levels from samples could be captured using the proposed growth system. This kind of growth system has been used to investigate short- and long-term responses to nitrogen depletion and nitrate re-addition in Arabidopsis [52].

Figure 2. Biomass of Arabidopsis seedlings grown under the developed cultivation system. (a) Visual phenotypes of Arabidopsis seedlings grown in sterilized Milli-Q water (left) or sterile liquid culture in Murashige and Skoog (MS) medium and 1\% sucrose (right) after seven-day cultivation. Twenty seedlings were put in an HS vial. Scale bar, $5 \mathrm{~cm}$. (b) Biomass of Arabidopsis seedlings grown in vials sealed by a silicon/polytetrafluoroethylene septa (PTFE) cap and a MilliSeal cap ( $n=10$ for each condition). Significance was determined by $t$-test ( $p$-value: $* * p<0.005$; *** $p<0.0005$ ). Abbreviations: DW, dry weight; W, week.
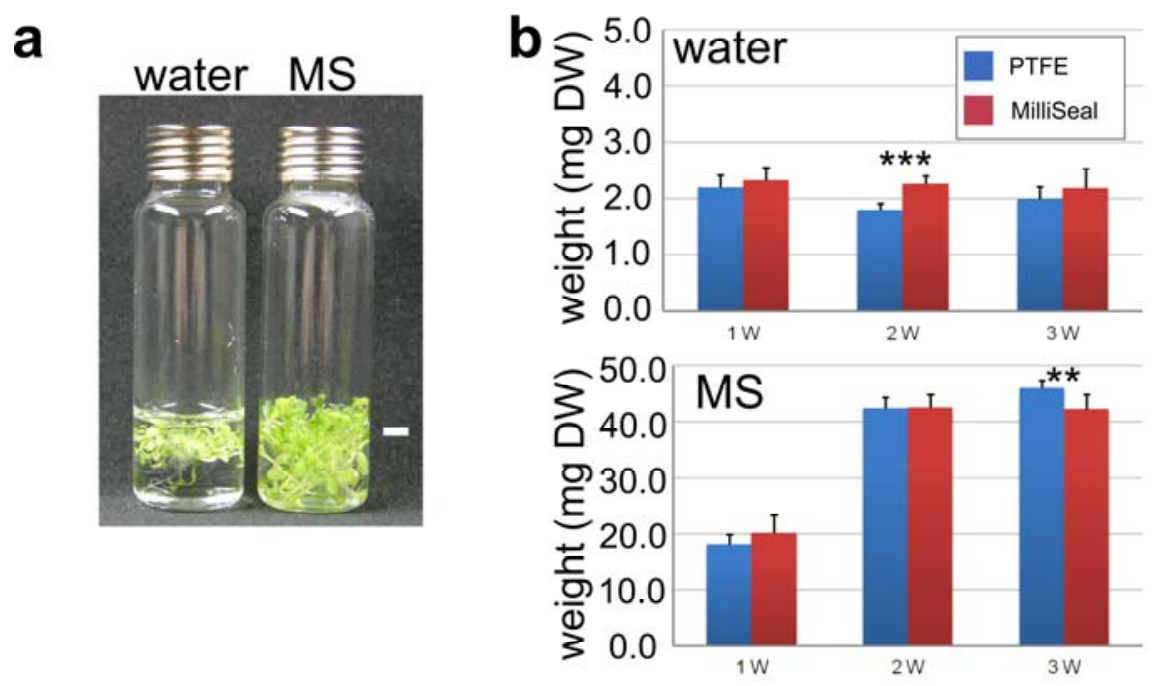


\subsection{Optimization of HS Collection for VOC Profiling}

VOCs in the HS of Arabidopsis seedlings could be collected directly by transferring the HS vials to the autosampler. To optimize extraction and collection of the HS grown in water and MS conditions, we first used three extraction methods (Figure 1). Next, we used nine fibers to evaluate the collection efficiency of the VOCs. By using the selected fiber, we optimized incubation conditions by changing the incubation time and temperature to collect the HS from Arabidopsis seedlings.

\subsubsection{HS Extraction}

Since the seedlings were alive in each vial just before HS collection, it was necessary to inactivate certain enzymes, such as lipoxygenase and hydroperoxide lyases, which contribute to VOC production. We compared each total ion chromatogram (TIC) obtained by applying the three extraction methods for the water and MS samples. $\mathrm{CaCl}_{2}$ is commonly used to stop enzyme activity, as well as to promote the release of VOCs to the HS from the plant tissues [53]. Ethylene-diamine-tetra-acetic acid (EDTA) can also inhibit enzyme activity in the cell $[54,55]$. In addition, the combined use of EDTA and $\mathrm{CaCl}_{2}$ provides highly reproducible VOC emission from tomato fruit [39], which is important when conducting metabolomic analysis. Therefore, we added EDTA, followed by $\mathrm{CaCl}_{2}$, to each $\mathrm{HS}$ vial for HS extraction. To validate the HS extraction method using EDTA and $\mathrm{CaCl}_{2}$, we heated other sets of HS vials to $80{ }^{\circ} \mathrm{C}$ to quench the enzyme activity in the seedlings. HS-VOCs emitted from untreated seedlings were also collected. We used Arabidopsis seedlings grown in water and MS conditions and performed HS-SPME-GC-TOF-MS analysis on these samples. A distinct pattern was seen in TICs of HS-VOCs, which were extracted by (i) adding EDTA solution followed by $\mathrm{CaCl}_{2}$ and (ii) heating at $80^{\circ} \mathrm{C}$. TICs of HS-VOCs from treatment (ii) and those emitted from the untreated seedlings looked similar, particularly when the samples were grown in water (Supplementary Figure S1). Peaks detected in samples from treatment (i) outnumbered other methods (Supplementary Figure S2). On the basis of the results, we chose to use a combination of EDTA and $\mathrm{CaCl}_{2}$ to extract HS-VOCs for further analysis.

\subsubsection{SPME Fiber Selection}

Many types of SPME fibers are available, which is one of the advantages of choosing the SPME method to collect the HS. According to the molecular weight and polarity of the analytes, an appropriate fiber should be chosen. We tested nine fibers to collect the HS from seedlings grown under the two conditions (Figure 2). Figure 3 a presents representative TICs of HS-VOCs collected by using a 7- $\mu \mathrm{m}$-thick layer of polydimethylsiloxane (PDMS), 100- $\mu \mathrm{m}$ PDMS, $75-\mu \mathrm{m}$ carboxen/PDMS (C/P) and carbowax-polyethylene glycol (C-PEG) fibers. Many peaks were eluted later in the TIC when we used a 7- $\mu \mathrm{m}$ PDMS fiber, as well as a C-PEG fiber (Figure 2a, top and bottom). This is because a 7- $\mu \mathrm{m}$ PDMS fiber favors non-polar and high-molecular-weight compounds or semi-volatile compounds, while a PEG fiber prefers to collect alcohols and polar compounds. On the other hand, a 75- $\mu \mathrm{m} \mathrm{C/P}$ fiber can absorb gases and low-molecular-weight compounds, which are eluted early in the TIC (Figure 3a). The VOC profiles obtained using a 100- $\mu \mathrm{m}$ PDMS fiber were highly scattered in the TIC (Figure 3a). 
Figure 3. Collection efficiency of HS-VOCs by using various solid phase microextraction (SPME) fibers. (a) Total ion chromatograms (TICs) of VOC profiles collected by using a 7- $\mu \mathrm{m}$ polydimethylsiloxane (PDMS) fiber (P 7), a 100- $\mu \mathrm{m}$ PDMS fiber (P 100), a 75- $\mu \mathrm{m}$ carboxen/PDMS fiber (C/P 75) and a carbowax-polyethylene glycol fiber (C-PEG). X-axis represents retention time, while the $\mathrm{Y}$-axis shows peak intensity. (b) Principal component analysis (PCA) score scatter plot of VOC profiles using nine SPME fibers (observation: samples, $n=36$; variables: annotated peaks, $n=99$ ). Four independent samples were analyzed using each SMPE fiber. HS-VOCs were collected from Arabidopsis seedlings grown in water and MS conditions ( $n=2$ each). (c) Venn diagram of the annotated peaks that showed significant changes in the profiles of (i) the $75-\mu \mathrm{m} \mathrm{C} / \mathrm{P}$ (blue), (ii) the 7- $\mu \mathrm{m}$ PDMS (yellow) and (iii) the C-PEG fiber collection (green) when compared to those collected by the 100- $\mu \mathrm{m}$ PDMS fiber. The number in the blue circle represents how many annotated peaks showed significant changes in the profiles from the HS-GC-TOF-MS analysis by using the $75-\mu \mathrm{m} \mathrm{C/P}$ fiber. The number in the yellow circle represents the number of significant peaks in the profile from the $7-\mu \mathrm{m}$ PDMS analysis. The number in the green circle represents the number of significant peaks in the HS profile from the C-PEG fiber collection. Control, the HS profile collected by the $100-\mu \mathrm{m}$ PDMS fiber (see Table 1). Abbreviation: PC, principal component.
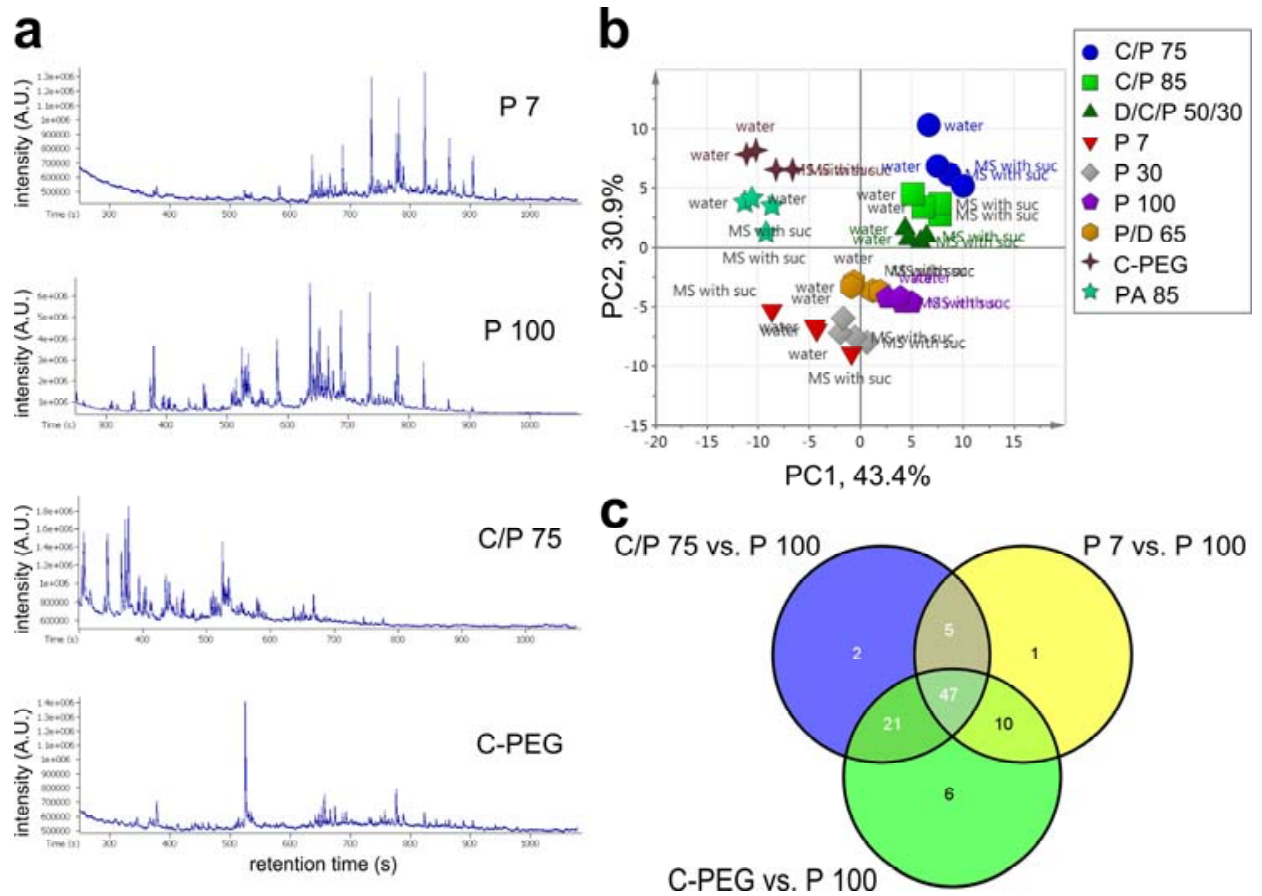

The HS-SPME-GC-TOF-MS analysis showed 437 peaks and their mass spectra. A total of 99 peaks were annotated as known VOCs or peaks with original IDs provided by VocBinBase [36] (Supplementary Table S1, S2). Principal component analysis (PCA) was applied to create a projection of multivariate data onto a space of lower dimensionality for visualization purposes (Figure $3 \mathrm{~b}$ ). The PCA score scatter plot showed clear separation in accordance with each fiber property. Often, SPME fibers coated with either 100- $\mu \mathrm{m}$ PDMS [42,53,56] or $65-\mu \mathrm{m}$-thick PDMS/divinylbenzene (DVB) $[39,57]$ are used as a first choice, because the former prefers to absorb non-polar metabolites, 
while the latter can extract short-chain polar compounds, e.g., alcohols and amines. The PCA score scatter plot illustrates that samples obtained by using a 100- $\mu \mathrm{m}$ PDMS fiber and those obtained by using a $65-\mu \mathrm{m}$ PDMS/DVB fiber were clustered very closely (Figure $3 b$ ). This suggests that the collection efficiency of these two fibers is probably very similar. We next evaluated the fiber specificity for the collected volatile substances in the HC of Arabidopsis seedlings by using the four representative fibers (75- $\mu \mathrm{m}$ C/P, 7- $\mu \mathrm{m}$ PDMS, 100- $\mu \mathrm{m}$ PDMS and C-PEG) that were chosen from the results of the PCA (Figure 3b). Among 99 annotated peaks, about half the peaks (47 peaks) could be commonly captured across the three fibers, unlike that in the case of those collected using the $100-\mu \mathrm{m}$ PDMS fiber (Figure 3c). This result shows that the major VOCs collected from the developed cultivation system were likely to be less polar in terms of physicochemical properties. On the other hand, there were a few of the fiber-specific peaks (Table 1). In particular, a C-PEG fiber could attract relatively polar compounds, such as alcohols. We selected a 100- $\mu \mathrm{m}$ PDMS fiber as the first choice.

Table 1. $\log _{2}$-fold changes in fiber-specific peaks collected using the $75-\mu \mathrm{m} \mathrm{C/P}, 7-\mu \mathrm{m}$ PDMS and C-PEG fibers, unlike that observed in the case of 100- $\mu \mathrm{m}$ PDMS fiber.

\begin{tabular}{|c|c|c|c|c|c|c|}
\hline Compound annotation & $\begin{array}{l}\log _{2}-\mathrm{FC} \\
(\mathrm{C} / \mathrm{P} 75 \text { vs. P 100) }\end{array}$ & FDR & $\begin{array}{l}\log _{2}-\mathrm{FC} \\
\text { (P } 7 \text { vs. P 100) }\end{array}$ & FDR & $\begin{array}{l}\log _{2}-\mathrm{FC} \\
\text { (C-PEG vs. P 100) }\end{array}$ & FDR \\
\hline Heptane, 5-ethyl-2-methyl- & 3.8 & 0.0000 & n.s. & & n.s. & \\
\hline Dodecane, 4,6-dimethyl- & 2.5 & 0.0046 & n.s. & & n.s. & \\
\hline 45514 & n.s. & & 4.6 & 0.0139 & n.s. & \\
\hline 1H-Indole, 1-methoxy- & n.s. & & n.s. & & 4.5 & 0.0090 \\
\hline 2-Undecanone & n.s. & & n.s. & & 4.4 & 0.0000 \\
\hline Undecanal & n.s. & & n.s. & & 3.7 & 0.0001 \\
\hline Biphenyl & n.s. & & n.s. & & 6.6 & 0.0000 \\
\hline Dodecanal & n.s. & & n.s. & & 3.9 & 0.0000 \\
\hline$n$-Tridecan-1-ol & n.s. & & n.s. & & 5.4 & 0.0010 \\
\hline
\end{tabular}

Significant, false discovery rate (FDR) of $<0.05$. Biological replicates, $n=4$ per fiber. Abbreviations: $\log _{2}$-FC, $\log _{2}$-fold change; C/P 75, a $75-\mu \mathrm{m} \mathrm{C/P}$ fiber; P 7 , a 7- $\mu \mathrm{m}$ PDMS fiber; CPEG, a C-PEG fiber; P 100, a 100- $\mu$ m PDMS fiber; n.s., not significant.

\subsubsection{Optimization of Incubation Conditions}

We used a 100- $\mu \mathrm{m}$ PDMS fiber and evaluated collection efficiencies by changing incubation conditions, times and temperatures. The PCA was applied to visualize relationships between incubation time or temperature and collection efficiency of volatiles (Figure 4). We first evaluated incubation time to optimize the collection efficiency of volatiles. We fixed the incubation temperature at $60{ }^{\circ} \mathrm{C}$ and then performed HS-SPME-GC-TOF-MS analysis by setting the incubation times at 10 , 20,30, 40, 50, 60, 70, 80 or 90 min (Figure 4a). Most of the samples were clustered together on the PCA scatter plot; however, samples incubated at 60 and $90 \mathrm{~min}$ showed relatively low reproducibility (Figure 4a, left). The PCA loading scatter plot demonstrated that longer incubation times resulted in more peaks than shorter incubation times did (Figure 4a, right). As metabolomics requires 
high-throughput analysis with high reproducibility, we chose $20 \mathrm{~min}$ as the optimum time for the incubation of the SPME vials.

Figure 4. Evaluation of incubation time (a) and temperature (b) to optimize collection efficiency of volatiles in the HS vials containing Arabidopsis seedlings grown in water and MS conditions. Left, PCA score scatter plot. Each symbol represents an independent sample. Right, PCA loading scatter plot. The gray dot shows an annotated peak. Four independent samples (two samples per condition) were analyzed per incubation time or temperature.

\section{a. Incubation time}
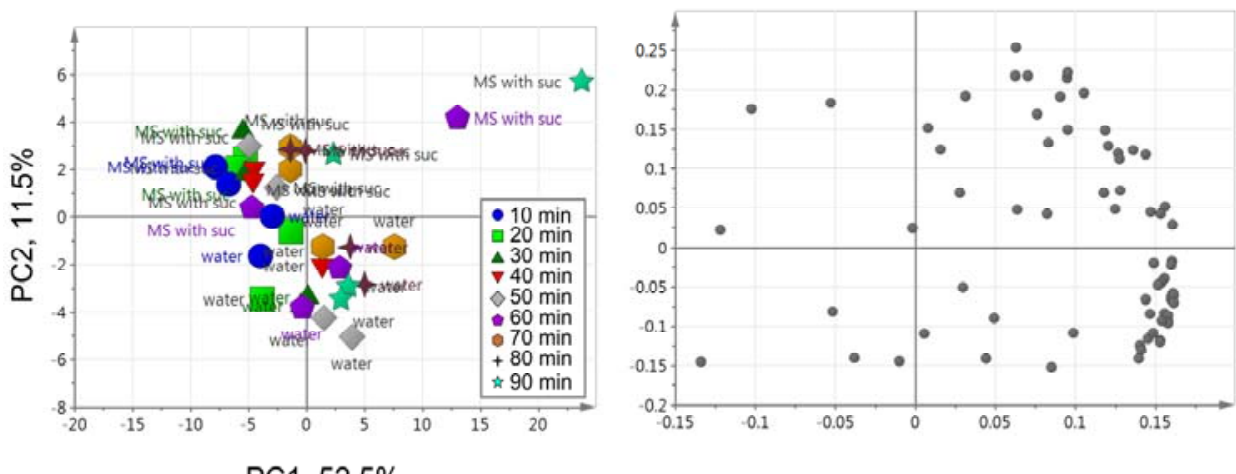

PC1, 52.5\%

\section{b. Incubation temperature}
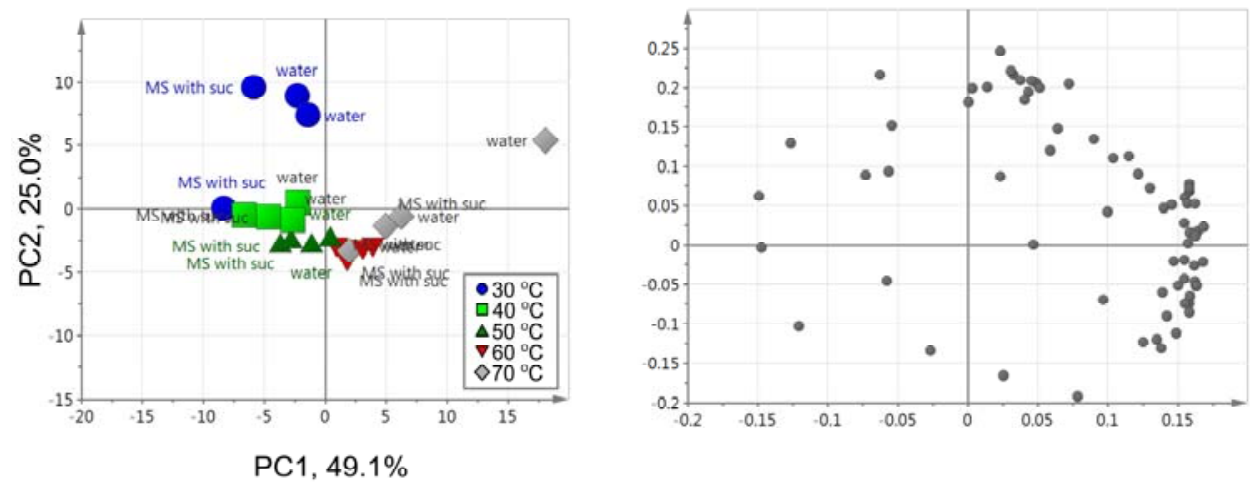

Next, we evaluated incubation temperature by setting it at $30^{\circ} \mathrm{C}, 40^{\circ} \mathrm{C}, 50{ }^{\circ} \mathrm{C}, 60{ }^{\circ} \mathrm{C}$ or $70{ }^{\circ} \mathrm{C}$. The HS-VOCs were collected for 20 min by SPME with a 100- $\mu \mathrm{m}$ PDMS fiber. The PCA indicated that the largest source of variation was the difference in incubation temperature and, in particular, the difference between incubation at $30^{\circ} \mathrm{C}$ and $70{ }^{\circ} \mathrm{C}$ (Figure $4 \mathrm{~b}$, left, PC1). Peaks were extracted at low and high incubation temperatures (Figure $4 \mathrm{~b}$, right), but samples incubated at $30{ }^{\circ} \mathrm{C}$ showed low reproducibility. On the other hand, the second source of variation could be explained by the differences between the samples incubated at $50{ }^{\circ} \mathrm{C}-70{ }^{\circ} \mathrm{C}$, and those at $30{ }^{\circ} \mathrm{C}$ (Figure $4 \mathrm{~b}$, left, PC2) include aliphatic alcohols, aliphatic/aromatic aldehydes, esters, ethers, hydrocarbons, including alkanes, ketones and terpenes [39,50,53]. Arabidopsis and Brassicaseae species can emit isothiocyanates and nitriles [58]. These VOCs have high vapor pressures and low-to-medium water solubility. Since some terpenes are unstable at high temperature [59,60], incubations must be performed under mild conditions. Therefore, the incubation temperature was set at $60{ }^{\circ} \mathrm{C}$. 


\subsection{VOC Profiling by Using a Developed Workflow - A Case Example}

We hypothesized that VOC profiles may differ when grown under the two nutrient conditions, because the biomass of the Arabidopsis seedlings grown in the MS condition was dramatically higher than that in water (Figure 1a,b). This implies that samples in water may be exposed to severe stress compared to those in MS. Using the optimized analytical conditions, we performed VOC profiling of Arabidopsis seedlings grown under the two conditions.

We obtained 263 peaks and their mass spectra by performing HS-SPME-GC-TOF-MS analysis. A total of 69 peaks were annotated as known VOCs or peaks with VocBinBase IDs (Supplementary Table S1, S3). To find the VOCs that contributed to differences between the VOC profiles of samples harvested from MS and those from water, bidirectional orthogonal projection onto latent structure-discriminant analysis (O2PLS-DA) were performed [61,62]. In this case, O2PLS-DA can extract as much of the class-separating (MS vs. water) variation as possible. The O2PLS-DA score scatter plot of samples grown under the two conditions showed a clear separation (Figure 5a). For example, we could detect the peak annotated as 1-isothiocyanato-heptane (Figure 5b), which is produced from aliphatic glucosinolates $[58,63]$. When considering the biosynthesis of isothiocyanates in Arabidopsis, the isothiocyanate would contain a branched chain rather than an odd-numbered chain [64]. GC-high-resolution (HR)-TOF-MS analysis has great potential in more reliably estimating the structure of such a peak.

The 39 VOCs were predicted to be discriminative metabolites by the O2PLS-DA loadings in the first Y-predictive component. Of these, 37 peaks tended to increase in water (e.g., calamenen-10-one), while the levels of two peaks tended to increase when the samples were grown in MS (e.g., 3-Buten-2-one, 4-(2,6,6-trimethyl-1-cyclohexen-1-yl)-, (E)-). 
Figure 5. O2PLS-DA score scatter plot (left) and loading plot (right) of VOC profiles elicited from Arabidopsis seedlings grown under the two different conditions. Each symbol represents an independent sample in the score scatter plot and an independent annotated peak in the loading plot. The levels of VOCs annotated as V126, V174 and V273 emitted from the samples grown in water (biological replicates, $n=10$ ) showed significant changes, when compared with those grown in MS (biological replicates, $n=10$ ) (see Table 2). The metabolites were extracted according to the first-weight vector $\left(\mathrm{w}^{*} 1\right)$ in addition to the $95 \%$ confidence intervals. A $p$-value (calculated by cross-validated predictive residuals, CV-ANOVA; $p \mathrm{cv}$ ) of $<0.01$ for the model was defined as significant. Abbreviations: pred comp, predictive component; ortho comp orthogonal component; HepICT, 1-isothiocyanato-heptane.

a

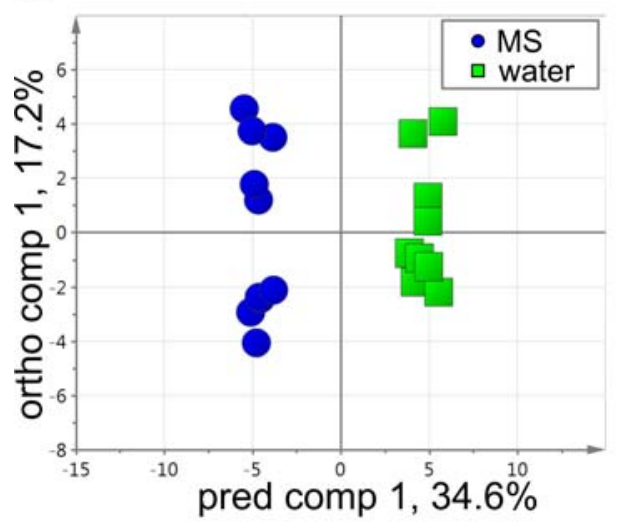

b

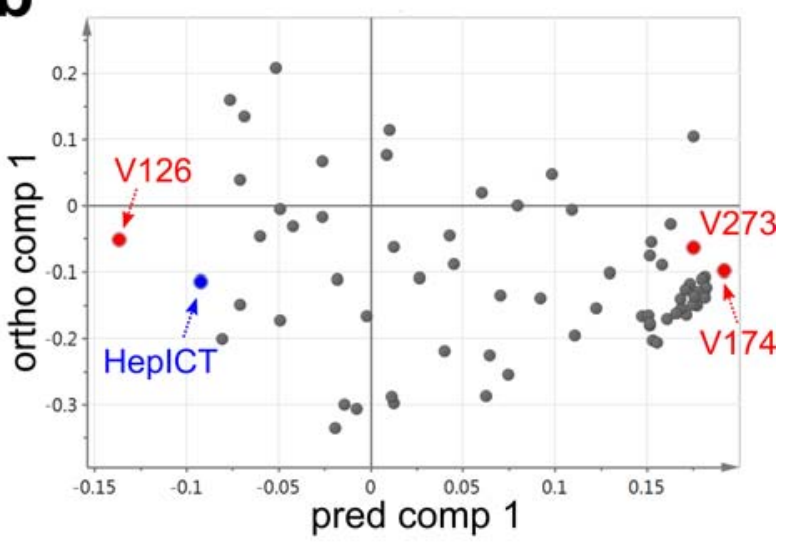

Subsequently, a $t$-test was applied to compare the two groups, MS vs. water (Table 2). The nine volatiles showed significant changes (false discovery rate $(\mathrm{FDR})$ of $<0.05$ ). A significant decrease was observed in the level of the VOC, called V126, when compared to that from samples grown in MS. The peak V126 annotated as $\beta$-ionone is known to be a flavor VOC emitted from various plant tissues $[65,66]$. $\beta$-ionone is produced via the enzymatic cleavage of carotenoid by dioxygenase in tomatoes [65]. The significant decrease in the level of $\beta$-ionone grown in water may be linked to carotenoid breakdown, since only the existence of water is very severe for the survival of Arabidopsis seedlings (Figure 1a,b). For instance, carotenoid content decreases during aging in tobacco leaves [67], and it also decreases when Arabidopsis leaves are shifted from a nutrient-sufficient medium to water [68].

The levels of eight volatiles, including unknown peaks with a VocBinBase ID, showed significant increases when compared to those from samples in MS. The peak, V154, is probably a column-derived peak, such as that of cyclooctasiloxanes, while the peak, V143, was classified as that of terpenoids according to the mass spectrum. There are many articles regarding V130 [69-72] and V174 in plant tissues [73-77]. However, the current method could not identify the V174 peak, because of the compound's orientation with respect to the benzene ring. 
Table 2. Significant changes in the levels of annotated volatiles.

\begin{tabular}{|c|c|c|c|}
\hline $\begin{array}{c}\text { Peak } \\
\text { ID }\end{array}$ & Compound annotation & $\begin{array}{c}\log _{2}-\mathrm{FC} \\
\text { (water vs. MS) }\end{array}$ & FDR \\
\hline V126 & 3-Buten-2-one, 4-(2,6,6-trimethyl-1-cyclohexen-1-yl)-, (E)- & -4.1 & 0.0000 \\
\hline V130 & Phenol, 2,4-bis(1,1-dimethylethyl)- & 3.4 & 0.0000 \\
\hline V143 & 47409 & 1.3 & 0.0160 \\
\hline V154 & 45485 & 2.1 & 0.0126 \\
\hline V172 & Hexadecanal & 1.3 & 0.0239 \\
\hline V173 & 1,1'-Biphenyl, 2,2',5,5'-tetramethyl- & 1.6 & 0.0069 \\
\hline V174 & calamenen-10-one & 1.7 & 0.0126 \\
\hline V194 & Phthalic acid, decyl isobutyl ester & 1.6 & 0.0307 \\
\hline V273 & 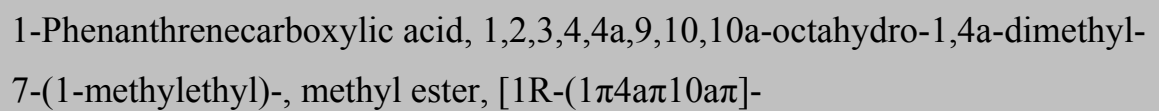 & 2.2 & 0.0261 \\
\hline
\end{tabular}

The changes were represented as the $\log _{2}$ - FC between the level of a volatile collected from samples grown in water and those grown in MS (significant, FDR $<0.05$; biological replicates, $n=10$ per condition).

By applying the developed method, we could discriminate between volatile compounds, including unknown peaks, from the two nutrient conditions, though they probably contained fiber- or column-derived compounds, such as biphenyl and silylated compounds. One of the current problems is in identifying the detected volatile peaks [78]. To accurately identify VOCs, authentic standards should be used in future studies. However, it is generally difficult to purchase authentic standards. As $\mathrm{GC} \times \mathrm{GC}$-TOF-MS-based profiling can improve the separation of VOCs, the profiling technique is expected to provide high-resolution VOC profiles. Another possible way to estimate VOC structures is to use GC-HR-TOF-MS, because the technique can provide us a highly accurate $\mathrm{m} / \mathrm{z}$ ratio with ultra-high resolution. Another big challenge of VOC profiling is to conduct absolute quantification of the detected peaks. We used one of the alkane standard peaks, i.e., tridecane, as an internal standard (IS) in the study. However, it is insufficient, because we can only cover alkene-like compounds to predict partition coefficient and phase ratio of volatile compounds in a HS vial. The use of various types of compounds, as well as fully-labeled alkanes as ISs, can increase to cover physicochemical properties of detected VOCs as much as possible. The approach may help to improve data quality in terms of semi-quantification analysis of VOCs.

\section{Experimental Section}

\subsection{Chemicals}

The $n$-alkane standard solutions C8-C20 were purchased from Fluka Chemical (Tokyo, Japan). Other chemicals were purchased from Nacalai Tesque (Kyoto, Japan) or Wako Pure Chemical Industries (Osaka, Japan). 


\subsection{Plant Material}

Wild-type Arabidopsis thaliana plants (accession Columbia (Col-0)) were used in this study. The plant seeds, which had been sterilized by sodium hypochlorite solution (Wako Pure Chemical Industries, Osaka, Japan), were stratified at $4{ }^{\circ} \mathrm{C}$ for 3 days. They were subsequently grown in MS medium (Wako Pure Chemical Industries) containing vitamins (Sigma-Aldrich, Tokyo, Japan; Lot, RNBB4051) with $0.8 \%$ agar and $1 \%$ sucrose at $\mathrm{pH}$ 5.8. Samples were exposed to fluorescent light under a 16-h light $\left(51-\mu \mathrm{mol} \cdot \mathrm{m}^{-2} \cdot \mathrm{s}^{-1}\right) / 8$-h dark cycle at $23{ }^{\circ} \mathrm{C}$ for 7 days in a growth chamber (MLR-350H; Sanyo, Osaka, Japan). Next, 20 seedlings were transferred into 20-ml HS vial (Supelco, Missouri, US) containing 5-mL of (i) sterile liquid culture of MS medium with $1 \%$ sucrose at pH 5.8 or (ii) sterilized Milli-Q water. The vials were closed with magnetic screw caps equipped with either Silicon/PTFE septa (AMR, Tokyo, Japan) or affixed with MilliSeals (EMD Millipore, Billerica, MA, USA). The seedlings were grown on a shaker (MMS-3010; Eyela, Tokyo, Japan) under a 16-h light $51-\mu \mathrm{mol} \cdot \mathrm{m}^{-2} \cdot \mathrm{s}^{-1}$ )/8-h dark cycle at $23{ }^{\circ} \mathrm{C}$ for 7,14 and 21 days. The shaker speed was set at $150 \mathrm{rpm}$ during the cultivation. The HS vials were directly used for HS collection via SPME.

\subsection{Headspace Collection and HS-SPME-GC-MS Analysis}

We used two methods and assayed to collect the HS of Arabidopsis seedlings after a 7 dayincubation. To quench enzyme activity in Arabidopsis, we treated the seedlings in the following manner: (i) a set of HS vials wrapped in aluminum foil were incubated at $80{ }^{\circ} \mathrm{C}$ for $30 \mathrm{~min}$ in a windy oven (WFO-600 ND; Eyela, Tokyo, Japan) and (ii) 0.25-M EDTA-NaOH water solution (pH 7.5) was added into another set of vials to attain a final EDTA concentration of $50 \mathrm{mM}$. Solid $\mathrm{CaCl}_{2}$ was then immediately added to a final concentration of $5 \mathrm{M}$ [39]. Next, $10-\mu \mathrm{L}$ of $n$-alkane standard solutions C8-20 $(0.8 \mathrm{mg} / \mathrm{L})$ was added to each vial as an internal standard. The vials were closed with magnetic screw-caps and then sonicated (US-108; NSD, Suwa, Japan) at a frequency of 38-Hz for 5 min. Vials without any treatment were prepared and used as controls.

Nine types of SPME fibers were purchased from Supelco (Supelco, PA, USA). A SPME fiber

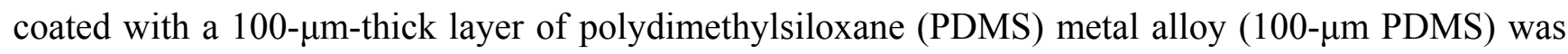
finally chosen for VOC profiling (see Results and Discussion section). SPME fibers were coated with (i) $30-\mu \mathrm{m}$ - or (ii) $7-\mu \mathrm{m}$-thick layers of PDMS-fused silica (FS) fiber/stainless steel (SS), (iii) $75-\mu \mathrm{m}$-thick layer of carboxen/polydimethylsiloxane (CAR/PDMS) FS/SS, (iv) 85- $\mu$ m-thick layer of CAR/PDMS StableFlex (SF) fiber/SS, (v) 65- $\mu$ m-thick layer of PDMS/divinylbenzene (DVB) $\mathrm{SF} / \mathrm{SS}$, (vi) 50/30- $\mu \mathrm{m}$-thick layer of DVB/CAR on PDMS (DVB/CAR/PDMS) SF/SS, (vii) 85 - $\mu \mathrm{m}$-thick layer of polyacrylate (PA 85) or (viii) carbowax-polyethylene glycol (C-PEG) for HS collection. Before the analysis, all fibers were conditioned at the appropriate conditioning temperature and time as shown in Table 3. Each fiber was exposed to the vial headspace for $20 \mathrm{~min}$ at $60{ }^{\circ} \mathrm{C}$ with continuous agitation.

After HS collection, the volatiles were thermally desorbed in splitless mode on a CTC CombiPAL autosampler (CTC analytics, Zwingen, Switzerland) connected to an Agilent 6890N gas chromatograph (Agilent Technologies, Wilmington, USA) for $0.1 \mathrm{~min}$ at the appropriate inlet 
temperature, as shown below. Each fiber was baked for $5 \mathrm{~min}$ by applying the appropriate conditioning temperature (see below).

Table 3. Conditioning temperature and time for the SPME fibers used in the study.

\begin{tabular}{|c|c|c|c|}
\hline SPME fiber & $\begin{array}{l}\text { Conditioning } \\
\text { temperature } \\
\left.{ }^{\circ} \mathrm{C}\right)\end{array}$ & $\begin{array}{l}\text { Conditioning } \\
\text { ( time }(\mathrm{h})\end{array}$ & $\begin{array}{l}\text { Inlet } \\
\text { temperature ( } \\
\left.{ }^{\circ} \mathrm{C}\right)\end{array}$ \\
\hline $100-\mu \mathrm{m}$ PDMS & 250 & 0.5 & 220 \\
\hline 30- $\mu \mathrm{m}$ PDMS & 250 & 0.5 & 220 \\
\hline 7- $\mu \mathrm{m}$ PDMS & 320 & 0.5 & 220 \\
\hline $85-\mu \mathrm{m}$ polyacrylate & 280 & 1 & 220 \\
\hline $60-\mu \mathrm{m}$ PEG & 240 & 0.5 & 220 \\
\hline 75- $\mu \mathrm{m}$ CAR/PDMS & 300 & 1 & 280 \\
\hline $85-\mu \mathrm{m}$ CAR/PDMS & 300 & 1 & 280 \\
\hline $65-\mu \mathrm{m}$ PDMS/DVB & 250 & 0.5 & 250 \\
\hline $50 / 30-\mu \mathrm{m}$ & 270 & 0.5 & 250 \\
\hline DVB/CAR/PDMS & & & \\
\hline
\end{tabular}

The capillary column used for the analysis was a $30-\mathrm{m} \times 0.25-\mathrm{mm}$ inner diameter fused-silica capillary column with a chemically bound $0.25-\mu \mathrm{L}$ film Rxi-5 Sil MS stationary phase (RESTEK, Bellefonte, USA) with a tandem connection to a fused silica tube $(1 \mathrm{~m}, 0.15 \mathrm{~mm})$. A mass spectrometer column change interface (ms NoVent-J; SGE, Yokohama, Japan) was used to prevent air and water from entering the MS during column change over. Helium was used as the carrier gas at a constant flow rate of $1.0 \mathrm{ml} / \mathrm{min}$. The temperature program started with a 2 min isothermal step at $50{ }^{\circ} \mathrm{C}$, followed by temperature ramping at $15^{\circ} \mathrm{C}$ to a final temperature of $260{ }^{\circ} \mathrm{C}$, which was then maintained for $2 \mathrm{~min}$. The transfer line to the mass spectrometer was set to $250{ }^{\circ} \mathrm{C}$. The TOF mass spectrometer was a Pegasus 4D MS system (Leco, MI, USA) with an EI source set to $200{ }^{\circ} \mathrm{C}$. The acceleration voltage was turned on after a solvent delay of $200 \mathrm{~s}$. Mass spectra were monitored with an acquisition rate of $30 \mathrm{spectra} / \mathrm{s}$ and over a mass-to-charge ratio range of $\mathrm{m} / \mathrm{z}=30-550$.

\subsection{Data Processing}

Non-processed MS data from GC-TOF-MS analysis were exported in NetCDF format, which was generated by the chromatography processing and mass spectral deconvolution software (Leco ChromaTOF version 2.32; LECO, MI, USA), to MATLAB 7.0 and MATLAB2011b (Mathworks, MA, USA), where all data pretreatment procedures, such as smoothing, alignment, time-window setting and hierarchical multivariate curve resolution (H-MCR), were carried out [79]. The resolved mass spectra were matched against the reference mass spectra by using the NIST mass spectral search program from the NIST/EPA/NIH mass spectral library (Data Version: NIST 08, Software Version 2.0) [34], Terpenoid library, Identification of Essential Oil Components by Gas Chromatography/Mass Spectrometry (the 4th Edition) commercial library [35] and VocBinbase [36]. Our custom software was also used for peak annotation written in Java. The metabolites were defined 
as annotated metabolites by comparison with RI values calculated by alkane standard mixtures (C8-20) and mass spectra from these three libraries. Peaks that showed a match factor of $\geq 800$ in the mass spectral library were selected for annotation. Data were normalized by dividing each peak area by the peak area of $n$-tridecane and metabolite identifiers were organized using MetMask [80].

\subsection{Data Analysis}

The two data matrices (observations: biological replicates, variables: annotated peaks) were used for statistical analyses, respectively (see Supplementary Tables S1-3). Multivariate analysis was performed using the SIMCA-P+13.0 software (Umetrics $\mathrm{AB}$, Umeå, Sweden), with $\log _{10}$ transformation and unit variance scaling. Metabolite profile data were $\log _{2}$ transformed and then statistically analyzed using the LIMMA package [81], which includes false discovery rate (FDR) correction for multiple testing (Benjamini and Hochberg, 1995) in the R environment for statistical computing (version 2.14.2). Analysis of variance testing of cross-validated predictive residuals (CV-ANOVA) was used as the significance test for assessing the reliability of O2PLS-DA models (significant, $p \mathrm{cv}<0.01$ ) [82].

\section{Conclusions}

VOC profiling using HP-SPME-GC-TOF-MS can distinguish between profiles with respect to each nutrient condition. More VOCs were expelled into the HS when Arabidopsis seedlings were exposed to conditions of limited nutrient supply, and the biomass of the seedlings showed severe growth reduction. Since we could easily change the nutrition conditions to grow the seedlings, we believe that the developed method will work well in investigating the assimilation of inorganic nutrients and production of VOCs, not only in Arabidopsis, but also in other plants. In addition, we can conduct metabolite profiling of Arabidopsis seedlings themselves grown using the cultivation method. This suggests a great potential for the integrated analysis of VOC profile data and metabolite profile data, not only to capture the changes in the levels of VOCs in plants grown under various environmental conditions, but also to understand the relationships among nutrition, biomass and metabolic networks involving primary and volatile metabolites.

\section{Acknowledgments}

We thank Tetsuya Sakurai and Kenji Akiyama for computational assistance and Koji Takano for technical assistance. This work was partly supported by the Japan Science and Technology Agency (JST), Strategic International Collaborative Research Program (SICORP).

\section{Conflict of Interest}

The authors declare no conflict of interest. 


\section{References and Notes}

1. Hakim, M.; Broza, Y.Y.; Barash, O.; Peled, N.; Phillips, M.; Amann, A.; Haick, H. Volatile Organic Compounds of Lung Cancer and Possible Biochemical Pathways. Chem. Rev. 2012, 112, 5949-5966.

2. Peled, N.; Hakim, M.; Bunn, P.A.; Miller, Y.E.; Kennedy, T.C.; Mattei, J.; Mitchell, J.D.; Hirsch, F.R.; Haick, H. Non-invasive Breath Analysis of Pulmonary Nodules. J. Thorac. Oncol. 2012, 7, $1528-1533$.

3. Buszewski, B.; Ligor, T.; Jezierski, T.; Wenda-Piesik, A.; Walczak, M.; Rudnicka, J. Identification of volatile lung cancer markers by gas chromatography-mass spectrometry: comparison with discrimination by canines. Anal. Bioanal. Chem. 2012, 404, 141-146.

4. Phillips, M.; Cataneo, R.N.; Saunders, C.; Hope, P.; Schmitt, P.; Wai, J. Volatile biomarkers in the breath of women with breast cancer. J. Breath Res. 2010, 4.

5. de Gennaro, G.; Dragonieri, S.; Longobardi, F.; Musti, M.; Stallone, G.; Trizio, L.; Tutino, M. Chemical characterization of exhaled breath to differentiate between patients with malignant plueral mesothelioma from subjects with similar professional asbestos exposure. Anal. Bioanal. Chem. 2010, 398, 3043-3050.

6. Abaffy, T.; Duncan, R.; Riemer, D.D.; Tietje, O.; Elgart, G.; Milikowski, C.; DeFazio, R.A. Differential Volatile Signatures from Skin, Naevi and Melanoma: A Novel Approach to Detect a Pathological Process. PLoS One 2010, 5.

7. Barash, O.; Peled, N.; Hirsch, F.R.; Haick, H. Sniffing the unique "odor print" of non-small-cell lung cancer with gold nanoparticles. Small 2009, 5, 2618-2624.

8. Gordon, S.M.; Szidon, J.P.; Krotoszynski, B.K.; Gibbons, R.D.; Oneill, H.J. Volatile organiccompounds in exhaled air from patients with lung-cancer. Clin. Chem. 1985, 31, 1278-1282.

9. Haick, H.; Hakim, M.; Patrascu, M.; Levenberg, C.; Shehada, N.; Nakhoul, F.; Abassi, Z. Sniffing chronic renal failure in rat model by an array of random networks of single-walled carbon nanotubes. Acs. Nano. 2009, 3, 1258-1266.

10. Peled, N.; Ionescu, R.; Nol, P.; Barash, O.; McCollum, M.; VerCauteren, K.; Koslow, M.; Stahl, R.; Rhyan, J.; Haick, H. Detection of volatile organic compounds in cattle naturally infected with Mycobacterium bovis. Sensor Actuat. B-Chem. 2012, 171, 588-594.

11. Leff, J.W.; Fierer, N. Volatile organic compound (VOC) emissions from soil and litter samples. Soil Biol. Biochem. 2008, 40, 1629-1636.

12. Ryu, C.M.; Farag, M.A.; Hu, C.H.; Reddy, M.S.; Kloepper, J.W.; Pare, P.W. Bacterial volatiles induce systemic resistance in Arabidopsis. Plant Physiol. 2004, 134, 1017-1026.

13. Ryu, C.M.; Farag, M.A.; Hu, C.H.; Reddy, M.S.; Wei, H.X.; Pare, P.W.; Kloepper, J.W. Bacterial volatiles promote growth in Arabidopsis. Proc. Natl. Acad. Sci. USA 2003, 100, 4927-4932.

14. Kai, M.; Haustein, M.; Molina, F.; Petri, A.; Scholz, B.; Piechulla, B. Bacterial volatiles and their action potential. Appl. Microbiol. Biot. 2009, 81, 1001-1012.

15. Weise, T.; Kai, M.; Gummesson, A.; Troeger, A.; von Reuss, S.; Piepenborn, S.; Kosterka, F.; Sklorz, M.; Zimmermann, R.; Francke, W.; Piechulla, B. Volatile organic compounds produced by the phytopathogenic bacterium Xanthomonas campestris pv. vesicatoria 85-10. Beilstein J. Org. Chem. 2012, 8, 579-596. 
16. Knudsen, J.T.; Eriksson, R.; Gershenzon, J.; Stahl, B. Diversity and distribution of floral scent. Botanical Rev. 2006, 72, 1-120.

17. Dudareva, N.; Negre, F.; Nagegowda, D.A.; Orlova, I. Plant volatiles: Recent advances and future perspectives. Crit Rev. Plant Sci. 2006, 25, 417-440.

18. D'Alessandro, M.; Turlings, T.C.J. Advances and challenges in the identification of volatiles that mediate interactions among plants and arthropods. Analyst 2006, 131, 24-32.

19. Loreto, F.; Schnitzler, J.P. Abiotic stresses and induced BVOCs. Trends Plant Sci. 2010, 15, 154166.

20. Kegge, W.; Pierik, R. Biogenic volatile organic compounds and plant competition. Trends Plant Sci. 2010, 15, 126-132.

21. Dicke, M.; van Loon, J.J.; Soler, R. Chemical complexity of volatiles from plants induced by multiple attack. Nat. Chem. Biol. 2009, 5, 317-324.

22. Tholl, D.; Boland, W.; Hansel, A.; Loreto, F.; Rose, U.S.; Schnitzler, J.P. Practical approaches to plant volatile analysis. Plant J. 2006, 45, 540-560.

23. Badoil, L.; Benanou, D. Characterization of volatile and semivolatile compounds in waste landfill leachates using stir bar sorptive extraction-GC/MS. Anal. Bioanal. Chem. 2009, 393, 1043-1054.

24. Tredoux, A.; de Villiers, A.; Majek, P.; Lynen, F.; Crouch, A.; Sandra, P. Stir bar sorptive extraction combined with GC-MS analysis and chemometric methods for the classification of south African wines according to the volatile composition. J. Agric. Food Chem. 2008, 56, 42864296.

25. Ridgway, K.; Lalljie, S.P.D.; Smith, R.M. Comparison of in-tube sorptive extraction techniques for non-polar volatile organic compounds by gas chromatography with mass spectrometric detection. J. Chromatogr. A 2006, 1124, 181-186.

26. Rasanen, I.; Viinamaki, J.; Vuori, E.; Ojanpera, I. Headspace In-Tube Extraction Gas Chromatography-Mass Spectrometry for the Analysis of Hydroxylic Methyl-Derivatized and Volatile Organic Compounds in Blood and Urine. J. Anal. Toxicol. 2010, 34, 113-121.

27. Tisch, U.; Haick, H. Arrays of chemisensitive monolayer-capped metallic nanoparticles for diagnostic breath testing. Rev. Chem. Eng. 2010, 26, 171-179.

28. Tisch, U.; Haick, H. Nanomaterials for cross-reactive sensor arrays. Mrs Bull. 2010, 35, 797-803.

29. Qualley, A.V.; Dudareva, N. Metabolomics of plant volatiles. Methods Mol. Biol. 2009, 553, 329343.

30. Saito, K.; Matsuda, F. Metabolomics for functional genomics, systems biology, and biotechnology. Annu. Rev. Plant Biol. 2010, 61, 463-489.

31. Roessner, U.; Willmitzer, L.; Fernie, A.R. Metabolic profiling and biochemical phenotyping of plant systems. Plant Cell. Rep. 2002, 21, 189-196.

32. Fiehn, O.; Kopka, J.; Dormann, P.; Altmann, T.; Trethewey, R.N.; Willmitzer, L. Metabolite profiling for plant functional genomics. Nat. Biotechnol. 2000, 18, 1157-1161.

33. Veriotti, T.; Sacks, R. High speed GC/MS of gasoline-range hydrocarbon compounds using a pressure-tunable column ensemble and time-of-flight detection. Anal. Chem. 2000, 72, 3063-3069. 
34. Stein, S.E.; Ausloos, P.; Clifton, C.L.; Klassen, J.K.; Lias, S.G.; Mikaya, A.I.; Sparkman, O.D.; Tchekhovskoi, D.V.; Zaikin, V.; Zhu, D. Evaluation of the NIST/EPA/NIH Mass Spectral Library. Abstr. Pap. Am. Chem. Soc. 1999, 218, U368-U368.

35. Adams, R.P. Identification of Essential Oil Components by Gas Chromatography/Quadrupole Mass Spectroscopy. 4th ed.; Allured Pub. Corporation: Carol Stream, IL, USA, 2007.

36. Skogerson, K.; Wohlgemuth, G.; Barupal, D.K.; Fiehn, O. The volatile compound BinBase mass spectral database. BMC Bioinformatics 2011, 12, 321.

37. Wagner, C.; Sefkow, M.; Kopka, J. Construction and application of a mass spectral and retention time index database generated from plant GC/EI-TOF-MS metabolite profiles. Phytochemistry 2003, 62, 887-900.

38. Aprea, E.; Gika, H.; Carlin, S.; Theodoridis, G.; Vrhovsek, U.; Mattivi, F. Metabolite profiling on apple volatile content based on solid phase microextraction and gas-chromatography time of flight mass spectrometry. J. Chromatogr. A 2011, 1218, 4517-4524.

39. Tikunov, Y.; Lommen, A.; de Vos, C.H.; Verhoeven, H.A.; Bino, R.J.; Hall, R.D.; Bovy, A.G. A novel approach for nontargeted data analysis for metabolomics. Large-scale profiling of tomato fruit volatiles. Plant Physiol. 2005, 139, 1125-1137.

40. Tikunov, Y.M.; de Vos, R.C.; Gonzalez Paramas, A.M.; Hall, R.D.; Bovy, A.G. A role for differential glycoconjugation in the emission of phenylpropanoid volatiles from tomato fruit discovered using a metabolic data fusion approach. Plant Physiol. 2010, 152, 55-70.

41. Roze, L.V.; Chanda, A.; Laivenieks, M.; Beaudry, R.M.; Artymovich, K.A.; Koptina, A.V.; Awad, D.W.; Valeeva, D.; Jones, A.D.; Linz, J.E. Volatile profiling reveals intracellular metabolic changes in Aspergillus parasiticus: veA regulates branched chain amino acid and ethanol metabolism. BMC Biochem. 2010, 11, 33.

42. Abel, C.; Clauss, M.; Schaub, A.; Gershenzon, J.; Tholl, D. Floral and insect-induced volatile formation in Arabidopsis lyrata ssp petraea, a perennial, outcrossing relative of A.thaliana. Planta 2009, 230, 1-11.

43. Aharoni, A.; Giri, A.P.; Deuerlein, S.; Griepink, F.; de Kogel, W.J.; Verstappen, F.W.; Verhoeven, H.A.; Jongsma, M.A.; Schwab, W.; Bouwmeester, H.J. Terpenoid metabolism in wild-type and transgenic Arabidopsis plants. Plant Cell. 2003, 15, 2866-2884.

44. Chen, F.; Tholl, D.; D'Auria, J.C.; Farooq, A.; Pichersky, E.; Gershenzon, J. Biosynthesis and emission of terpenoid volatiles from Arabidopsis flowers. Plant Cell. 2003, 15, 481-494.

45. Salas, J.J.; Garcia-Gonzalez, D.L.; Aparicio, R. Volatile compound biosynthesis by green leaves from an Arabidopsis thaliana hydroperoxide lyase knockout mutant. J. Agric. Food Chem 2006, 54, 8199-8205.

46. Huang, M.S.; Abel, C.; Sohrabi, R.; Petri, J.; Haupt, I.; Cosimano, J.; Gershenzon, J.; Tholl, D. Variation of Herbivore-Induced Volatile Terpenes among Arabidopsis Ecotypes Depends on Allelic Differences and Subcellular Targeting of Two Terpene Synthases, TPS02 and TPS03. Plant Physiol 2010, 153, 1293-1310.

47. Lee, S.; Badieyan, S.; Bevan, D.R.; Herde, M.; Gatz, C.; Tholl, D. Herbivore-induced and floral homoterpene volatiles are biosynthesized by a single P450 enzyme (CYP82G1) in Arabidopsis. Proc. Natl. Acad. Sci. USA 2010, 107, 21205-21210. 
48. Snoeren, T.A.; Kappers, I.F.; Broekgaarden, C.; Mumm, R.; Dicke, M.; Bouwmeester, H.J. Natural variation in herbivore-induced volatiles in Arabidopsis thaliana. J. Exp. Bot. 2010, 61, 3041-3056.

49. Huang, M.; Sanchez-Moreiras, A.M.; Abel, C.; Sohrabi, R.; Lee, S.; Gershenzon, J.; Tholl, D. The major volatile organic compound emitted from Arabidopsis thaliana flowers, the sesquiterpene (E)- $\beta$-caryophyllene, is a defense against a bacterial pathogen. New Phytologist 2012, 193, 997-1008.

50. Farag, M.A.; Ryu, C.M.; Sumner, L.W.; Pare, P.W. GC-MS SPME profiling of rhizobacterial volatiles reveals prospective inducers of growth promotion and induced systemic resistance in plants. Phytochemistry 2006, 67, 2262-2268.

51. Kusano, M.; Fukushima, A.; Arita, M.; Jonsson, P.; Moritz, T.; Kobayashi, M.; Hayashi, N.; Tohge, T.; Saito, K. Unbiased characterization of genotype-dependent metabolic regulations by metabolomic approach in Arabidopsis thaliana. BMC Syst. Biol. 2007, 1, 53.

52. Scheible, W.R.; Morcuende, R.; Czechowski, T.; Fritz, C.; Osuna, D.; Palacios-Rojas, N.; Schindelasch, D.; Thimm, O.; Udvardi, M.K.; Stitt, M. Genome-wide reprogramming of primary and secondary metabolism, protein synthesis, cellular growth processes, and the regulatory infrastructure of Arabidopsis in response to nitrogen. Plant Physiol. 2004, 136, 2483-2499.

53. Verdonk, J.C.; Ric de Vos, C.H.; Verhoeven, H.A.; Haring, M.A.; van Tunen, A.J.; Schuurink, R.C. Regulation of floral scent production in petunia revealed by targeted metabolomics. Phytochemistry 2003, 62, 997-1008.

54. Mandelli, J.; Wajner, A.; Pires, R.; Giugliani, R.; Coelho, J.C. Effect of CuCl2, NaCl and EDTA on the enzyme alpha-L-iduronidase in the plasma of normal individuals and heterozygotes for MPS I. Clinica Chimica Acta 2002, 318, 83-89.

55. Yang, W.C.; Schultz, R.D. Inhibitory Effect of Ethylenediaminetetraacetate (Edta) on the Porcine Lymphocyte-Response to Mitogens and Reversal of the Effect with Metal-Ions. Biol. Trace Elem. Res. 1982, 4, 175-182.

56. Ortiz-Serrano, P.; Gil, J.V. Quantitation of free and glycosidically bound volatiles in and effect of glycosidase addition on three tomato varieties (Solanum lycopersicum L.). J. Agric. Food Chem. 2007, 55, 9170-9176.

57. Obara, N.; Hasegawa, M.; Kodama, O. Induced volatiles in elicitor-treated and rice blast fungusinoculated rice leaves. Biosci. Biotechnol. Biochem. 2002, 66, 2549-2559.

58. Rohloff, J.; Bones, A.M. Volatile profiling of Arabidopsis thaliana - Putative olfactory compounds in plant communication. Phytochemistry 2005, 66, 1941-1955.

59. Agger, S.A.; Lopez-Gallego, F.; Hoye, T.R.; Schmidt-Dannert, C. Identification of sesquiterpene synthases from Nostoc punctiforme PCC 73102 and Nostoc sp. strain PCC 7120. J. Bacteriol. 2008, 190, 6084-6096.

60. Quintana, A.; Reinhard, J.; Faure, R.; Uva, P.; Bagneres, A.G.; Massiot, G.; Clement, J.L. Interspecific variation in terpenoid composition of defensive secretions of European Reticulitermes termites. J. Chem. Ecol. 2003, 29, 639-652.

61. Bylesjo, M.; Rantalainen, M.; Cloarec, O.; Nicholson, J.K.; Holmes, E.; Trygg, J. OPLS discriminant analysis: combining the strengths of PLS-DA and SIMCA classification. J. Chemom. 2006, 20, 341-351. 
62. Trygg, J.; Holmes, E.; Lundstedt, T. Chemometrics in metabonomics. J. Proteome Res. 2007, 6, 469-479.

63. Vercammen, J.; Pham-Tuan, H.; Arickx, I.; Van der Straeten, D.; Sandra, P. Monitoring of isothiocyanates emanating from Arabidopsis thaliana upon paraquat spraying. J. Chromatogr. A 2001, 912, 127-134.

64. Lambrix, V.; Reichelt, M.; Mitchell-Olds, T.; Kliebenstein, D.J.; Gershenzon, J. The Arabidopsis epithiospecifier protein promotes the hydrolysis of glucosinolates to nitriles and influences Trichoplusia ni herbivory. Plant Cell 2001, 13, 2793-2807.

65. Simkin, A.J.; Schwartz, S.H.; Auldridge, M.; Taylor, M.G.; Klee, H.J. The tomato carotenoid cleavage dioxygenase 1 genes contribute to the formation of the flavor volatiles beta-ionone, pseudoionone, and geranylacetone. Plant J. 2004, 40, 882-892.

66. Caja, M.D.; Preston, C.; Kempf, M.; Schreier, P. Flavor authentication studies of alpha-ionone, beta-ionone, and alpha-ionol from various sources. J. Agr. Food Chem. 2007, 55, 6700-6704.

67. Prochazkova, D.; Haisel, D.; Wilhelmova, N. Content of carotenoids during ageing and senescence of tobacco leaves with genetically modulated life-span. Photosynthetica 2009, 47, 409-414.

68. Thomas, H.; deVilliers, L. Gene expression in leaves of Arabidopsis thaliana induced to senesce by nutrient deprivation. J. Exp. Bot. 1996, 47, 1845-1852.

69. Al-Shwyeh, H.A.; Mirghani, M.E.S.; Jamal, P. Antibacterial activity of Malaysian mango kernel. Afr J. Biotechnol. 2011, 10, 18739-18748.

70. Miklosy, E.; Kerenyi, Z. Comparison of the volatile aroma components in noble rotted grape berries from two different locations of the Tokaj wine district in Hungary. Anal. Chim. Acta 2004, $513,177-181$.

71. Nikbakhtzadeh, M.R.; Tirgari, S.; Fakoorziba, M.R.; Alipour, H. Two volatiles from the venom gland of the Samsum ant, Pachycondyla senaarensis. Toxicon. 2009, 54, 80-82.

72. Sathya, A.; Ramasubramaniaraja, R.; Brindha, P. Pharmacognostical, phytochemical and GC-MS investigation of successive extracts of Gymnema syvestre R.Br. J. Pharm. Res. 2010, 3, 984-987.

73. Maggi, F.; Lucarini, D.; Tirillini, B.; Vittori, S.; Sagratini, G.; Papa, F. Essential oil composition of Ephedra nebrodensis Tineo ex Guss. subsp. nebrodensis from central Italy. J. Essent. Oil Res. 2010, 22, 354-357.

74. Velasco-Negueruela, A.; Perez-Alonso, M.J.; Perez de Paz, P.L.; Pala-Paul, J.; Sanz, J. Analysis by gas chromatography-mass spectrometry of the volatiles from the fruits of Ammodaucus leucotrichus subsp. leucotrichus and subsp. nanocarpus grown in North Africa and the Canary Islands, respectively. J. Chromatogr. A 2006, 1108, 273-275.

75. Radulovic, N.; Dekic, M.; Stojanovic-Radic, Z.; Palic, R. Volatile constituents of Erodium cicutarium (L.) L' H,rit. (Geraniaceae). Centr Eur J. Biol. 2009, 4, 404-410.

76. Silva, D.R.; Endo, E.H.; Dias, B.P.; Nakamura, C.V.; Svidzinski, T.I.E.; de Souza, A.; Young, M.C.M.; Ueda-Nakamura, T.; Cortez, D.A.G. Chemical Composition and Antimicrobial Properties of Piper ovatum Vahl. Molecules 2009, 14, 1171-1182.

77. Chaverri, C.; Diaz, C.; Ciccio, J.F. Chemical Analysis of Essential Oils from Ocotea gomezii WC Burger and Ocotea morae Gomez-Laur. (Lauraceae) Collected at "Reserva Biologica Alberto M. 
Brenes" in Costa Rica and their Cytotoxic Activity on Tumor Cell Lines. J. Braz Chem. Soc. 2011, 22, S1-S4.

78. Fiehn, O.; Robertson, D.; Griffin, J.; van der Werf, M.; Nikolau, B.; Morrison, N.; Sumner, L.W.; Goodacre, R.; Hardy, N.W.; Taylor, C.; Fostel, J.; Kristal, B.; Kaddurah-Daouk, R.; Mendes, P.; van Ommen, B.; Lindon, J.C.; Sansone, S.A. The metabolomics standards initiative (MSI). Metabolomics 2007, 3, 175-178.

79. Jonsson, P.; Johansson, E.S.; Wuolikainen, A.; Lindberg, J.; Schuppe-Koistinen, I.; Kusano, M.; Sjostrom, M.; Trygg, J.; Moritz, T.; Antti, H. Predictive metabolite profiling applying hierarchical multivariate curve resolution to GC-MS data--a potential tool for multi-parametric diagnosis. $J$. Proteome Res. 2006, 5, 1407-1414.

80. Redestig, H.; Kusano, M.; Fukushima, A.; Matsuda, F.; Saito, K.; Arita, M. Consolidating metabolite identifiers to enable contextual and multi-platform metabolomics data analysis. BMC Bioinformatics 2010, 11, 214.

81. Smyth, G.K. Linear models and empirical bayes methods for assessing differential expression in microarray experiments. Stat. Appl Genet. Mol. Biol. 2004, 3, Article 3.

82. Eriksson, L.; Trygg, J.; Wold, S. CV-ANOVA for significance testing of PLS and OPLS (R) models. J. Chem. 2008, 22, 594-600.

(C) 2013 by the authors; licensee MDPI, Basel, Switzerland. This article is an open access article distributed under the terms and conditions of the Creative Commons Attribution license (http://creativecommons.org/licenses/by/3.0/). 